\title{
Travelling waves in discrete electrical lattice with nonlinear symmetric capacitor
}

\author{
Alain Bertrand Togueu Motcheyo ( $\square$ alain.togueu@univ-yaounde1.cm ) \\ University of Yaounde I: Universite de Yaounde I https://orcid.org/0000-0002-6918-9281 \\ Georges Julius Fezeu \\ University of Yaounde I: Universite de Yaounde I \\ Martin Siewe Siewe \\ University of Yaounde I: Universite de Yaounde I \\ Clémence Nono Dueyou Buckjohn \\ University of Yaounde I: Universite de Yaounde I \\ Clément Tchawoua \\ University of Yaounde I: Universite de Yaounde I
}

\section{Research Article}

Keywords: Electrical transmission lines, Waves and wave propagation, Soliton

Posted Date: January 24th, 2022

DOI: https://doi.org/10.21203/rs.3.rs-1206297/v1

License: (9) This work is licensed under a Creative Commons Attribution 4.0 International License.

Read Full License 


\title{
Travelling waves in discrete electrical lattice with nonlinear symmetric capacitor
}

\author{
G. J. Fezeu - A. B. Togueu Motcheyo* - M. Siewe Siewe - C. Nono \\ Dueyou Buckjohn · C. Tchawoua
}

Received: date / Accepted: date

\begin{abstract}
We study the propagation of voltage in a model of the conventional right-handed transmission line with a nonlinear symmetric capacitor. Applying the quasidiscrete approximation to the nonlinear voltage equation of the line, we derive a nonlinear Schrödinger equation and find the bright and dark solutions which are used as the initial condition for the integration of the nonlinear lattice model. The full integration of the lattice shows the propagation of the nonlinear voltage in the right-hand side and its robustness in the time. The density energy of the lattice at each time has a node around which bright voltage is localized while in the case of dark voltage, it has a node where it seems to drop to zero at the soliton centre.
\end{abstract}

Keywords Electrical transmission lines - Waves and wave propagation $\cdot$ Soliton

\section{Introduction}

The use of discrete nonlinear electrical transmission lines as a theoretical or experimental tool to investigate soliton properties has been the focus of consider-

A. B. Togueu Motcheyo

Department of Mechanical Engineering, Higher Technical Teacher's Training College (ENSET) Ebolowa, University of Yaounde I, P.O. Box 886, Ebolowa-Cameroon

* Corresponding author

E-mail: alain.togueu@univ-yaounde1.cm, abtogueu@yahoo.fr, alainbertrandtogueu@gmail.com Tel.: $+237675141621$

G. J. Fezeu, M. Siewe Siewe, C. Nono Dueyou Buckjohn, C. Tchawoua

Laboratory of Mechanics, Department of Physics, Faculty of Science, University of Yaounde I, P.O. Box 812, Yaounde, Cameroon able research since the pioneering works by Hirota and Suzuki $[1,2]$. The ability of solitons to propagate with small dispersion can be used as an effective means to transmit data, modulated as short pulses over long distances justify in part its grown interest. Solitons finds an application in many areas including fibre-optic communications, Bose-Einstein condensation, DNA doublestrand modelling and electronic circuits [3,4]. Nonlinear transmission lines (NLTLs) which is a part of the latter mentioned area have become extremely active in recent years. This is due in part of the fact that they can be characterized using inexpensive laboratory equipment such as function generators and oscilloscopes; the availability of strongly nonlinear circuit elements. NLTLs are used for parametric amplification and pulse generation [5], for the management of localized energy [6], for nerve model with self-excitable membrane $[7,8]$, for the studies of diatomic lattice solitons $[9,10]$ and as a model of Microtubule [11], for the study of double-negative metamaterial[12], just to mention some examples.

It is known that solitary wave results from the balance of dispersion and nonlinearity effects. In NLTLs the nonlinearities come generally from the voltage dependence of the capacitance. The nonlinear capacitor can be in the form of logarithmic function as in the Toda lattice [13-20]. Marquié et al. have observed experimentally localized mode the electrical lattice with this form of nonlinear capacitor. The exponential [6] or the results of the Taylor expansion of an experimentally double exponential [21] function has been proposed . In the case where the nonlinear capacitance is the voltages reverse-biased diode, its expression has an asymmetric quadratic expression as in refs. [22-27].

More recently, the fabrication, measurement, and modelling of radio-frequency, tunable interdigital capacitors with ferroelectric barium strontium titanate 
[28] have open the way for the new studies in the electrical line. The voltage-dependent, symmetric capacitance of the material developed allows to constructing nonlinear left-handed metamaterials electrical line which supports rogue wave [29] and bright breathers [30]. It is then natural to see the behavior of the conventional transmission with this new type of nonlinear capacitor. To explore this way is the main purpose of this paper.

In the next section, we present the theoretical model, the energy of the lattice, the linear and nonlinear analysis. Numerical results are presented in Section 3. In Section 4 we summarize our results and conclude.

\section{Mathematical description of the model}

\subsection{Model description}

Following Refs. [13-19], let us consider a unit cell of a dispersive nonlinear transmission line shown in Figure 1 as our model under investigation. Each unit cell, such as the nth one, contains a linear inductor $\mathrm{L}_{1}$ in the series branch, and a linear inductor $\mathrm{L}_{2}$ in parallel with a nonlinear capacitor $\mathrm{C}\left(\mathrm{V}_{\mathrm{n}}\right)$ in the shunt branch. $\mathrm{V}_{\mathrm{n}}(\mathrm{t})$ denotes the voltage across the capacitor which capacitance is $\mathrm{C}\left(\mathrm{V}_{\mathrm{n}}\right), \mathrm{I}_{\mathrm{n}}$ the current through the inductor in the series branch, $\mathrm{I}_{\mathrm{n}}^{\prime}$ the current through $\mathrm{C}\left(\mathrm{V}_{\mathrm{n}}\right)$ and $\mathrm{I}_{\mathrm{n}}$ the current through the inductor in the shunt branch. Applying the Kirchhoff's laws at the nth section leads to the following set of conservation laws of the current and the charge:

$L_{1} \frac{\partial I_{n}}{\partial t}=V_{n-1}-V_{n}$

$L_{2} \frac{\partial I_{n}^{\prime \prime}}{\partial t}=V_{n}$

$I_{n}^{\prime \prime}=I_{n}-I_{n-1}-I_{n}^{\prime} ;$

$I_{n}^{\prime}=\frac{\partial Q_{n}}{\partial t}$

where $\mathrm{Q}_{\mathrm{n}}$ denotes the charge in the nonlinear capacitor and allows to determine the type of nonlinearity within the lattice. The nonlinear capacitor can be in the form of Toda lattice [13-20] or its expression can be obtained from the Taylor expansion of an experimentally double exponential form [21]. In that case, the nonlinear capacitance of the diode varactor has an asymmetric quadratic expression as in the low voltages reversebiased diode [22-27]. The choice of charge here is motivated by the recent development of strongly nonlinear and voltage symmetric barium strontium titanate thin film capacitors and the charge along the capacitance is given in the following $[29,30]$ :

$Q_{n}=C\left(V_{n}\right) \cdot V_{n}=C_{0}\left(1+a V_{n}^{2}\right) \cdot V_{n}$,

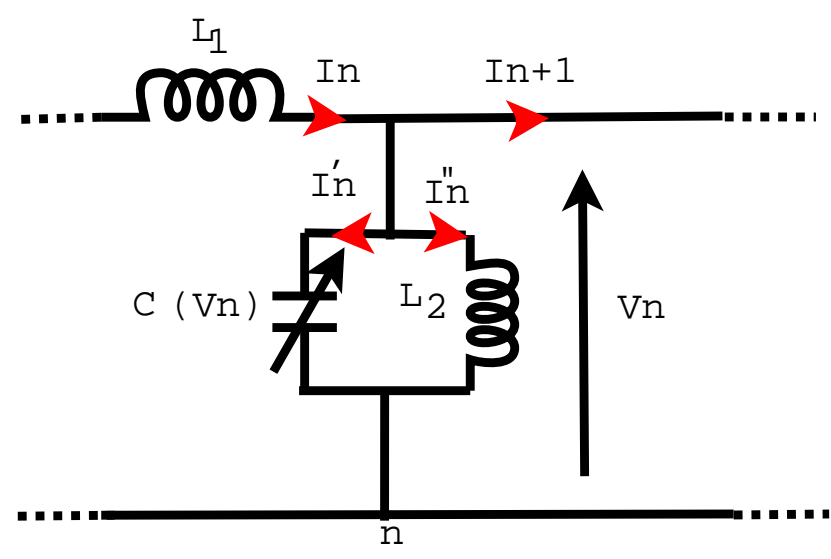

Fig. 1 Model for the unit-cell circuit of the lattice.

with $a=\frac{16}{9 V_{0}^{2}}$. Here $C_{0}$ is the zero bias capacitance and $V_{0}$ has the units of voltage. The form of the nonlinear charge (5) can be also found in split-ring resonator [32]. Using equations (1), (2), (3), (4) and (5), we can obtain the discrete voltage propagation equations of the lattice given by:

$\frac{\partial^{2}}{\partial t^{2}}\left(V_{n}+a V_{n}^{3}\right)=u_{0}^{2}\left(V_{n+1}+V_{n-1}-2 V_{n}\right)-\omega_{0}^{2} V_{n}$,

where $u_{0}^{2}=\frac{1}{L_{1} C_{0}}$ and $\omega_{0}^{2}=\frac{1}{L_{2} C_{0}}$ are the characteristic frequencies of the lattice. For the numerical experiment, the following parameters are used [14]

$\mathrm{L}_{1}=680 \mu \mathrm{H}, \quad \mathrm{L}_{2}=470 \mu \mathrm{H}, \quad \mathrm{C}_{0}=470 \mathrm{pF}, \quad \mathrm{V}_{0}=2 \mathrm{~V} .(7)$

\subsection{Energy of the lattice}

The local energy in the different capacitive and inductive elements will be derived following refs [30,31] as:

$E_{n}=\frac{1}{2} L_{1} I_{n}^{2}+\frac{1}{2} L_{2}\left(I_{n}^{\prime \prime}\right)^{2}+C_{0}\left(\frac{1}{2} V_{n}^{2}+\frac{3}{4} a V_{n}^{4}\right)$.

The current $\mathrm{I}_{\mathrm{n}}$ and $I_{n}^{\prime \prime}$ will be derived numerically using equations (1) and (2) respectively.

2.3 Linear analysis: linear dispersion and group velocity

Linear oscillation in the lattice with angular frequency $\omega$ and wave number $k$ are described by the following linear dispersion law:

$\omega^{2}=\omega_{0}^{2}+4 u_{0}^{2} \sin ^{2}\left(\frac{k}{2}\right)$.

The linear dispersion curve corresponding to equation (9) is depicted in figure 2. It appears a lower cutoff 


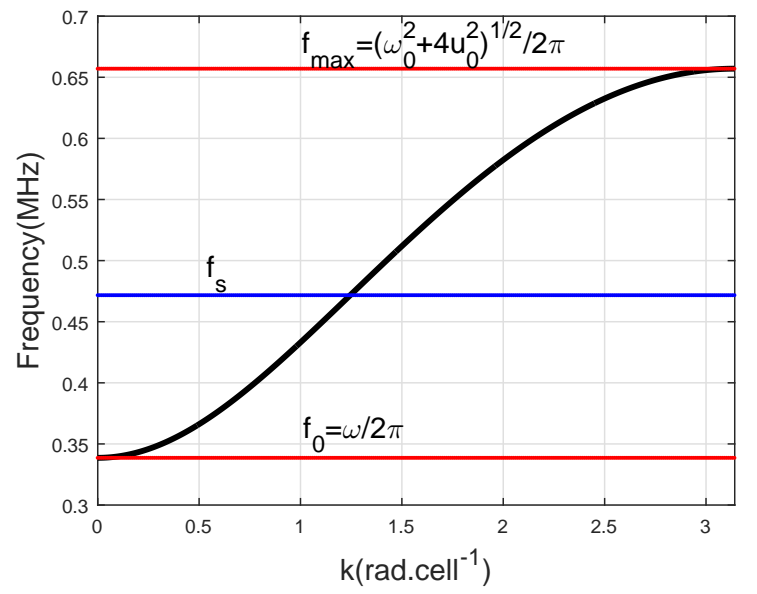

Fig. 2 Dispersion relation curves defined by Eq. (9).

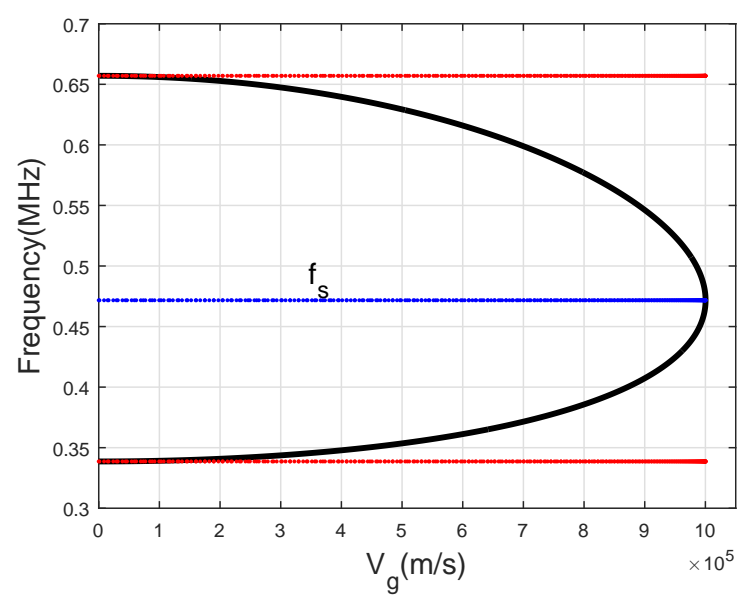

Fig. 3 Group velocity (10).

mode frequency $f_{0}=\omega_{0} / 2 \pi$ at $k=0$ and an upper frequency $f_{\max }=\left(\sqrt{\omega_{0}^{2}+4 u_{0}^{2}}\right) / 2 \pi$ at $k=\pi$. The latter is a consequence of the discretization of the lattice; this means that it does not exist in the continuum.

The group velocity relation associated with the wave packet is defined by

$V_{g}=\frac{\partial \omega}{\partial k}=\frac{u_{0}^{2}}{\omega} \sin (k)$.

Figure 3 depicts the variation of the frequency as a function of the group velocity. One can observe the positive values of the group velocity which imply the positive product of the phase velocity $V_{p h}=\frac{\omega}{k}$ and $V_{g}$. In fact, the transmission line is a conventional lattice then, adopts right-handed $(\mathrm{RH})$ behavior and the waves propagating in media are known as forward-waves [20, 33]. Different types of solitary waves in the RH transmission line can be found in the literature $[34,35, ?, 37$, 38 .

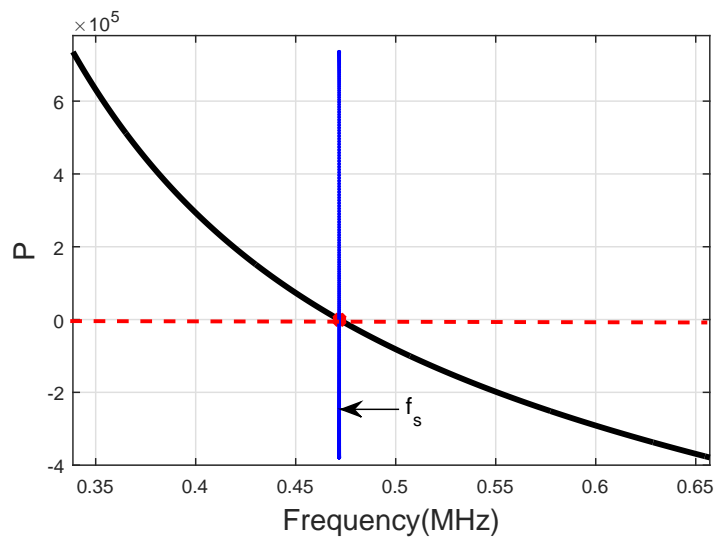

Fig. 4 Dispersion coefficient (13) as a function of the frequency.

2.4 The Nonlinear Schrödinger equation and solutions

Due to the symmetric nature of the capacitor, only one harmonic in the semi-discrete rotating-wave approximation can be used as the solutions of Eq. (6). Let us consider the solution as follows:

$\mathrm{V}_{\mathrm{n}}(\mathrm{t})=\epsilon \psi(\mathrm{x}, \tau) \exp \left(\mathrm{i} \theta_{\mathrm{n}}\right)+\epsilon \psi^{*}(\mathrm{x}, \tau) \exp \left(-\mathrm{i} \theta_{\mathrm{n}}\right)$,

where $\theta_{n}=k n-\omega t$ and $\psi$ stands for a complex unknown envelope function depending on the slow scales $x=\epsilon\left(n-V_{g} t\right)$ and $\tau=\epsilon^{2} t$. Inserting expression (11) in (6), we obtain the dispersion relation (9) and the group velocity (10) by keeping term of $\epsilon \exp (-i \omega t)$ and $\epsilon^{2} \exp (-i \omega t)$ respectively. Finally, we obtain a nonlinear evolution equation for the unknown function $\psi$ namely, the NLS equation by keeping the terms of order $\epsilon^{3} \exp (-i \omega t)$ as follows:

$\mathrm{i} \frac{\partial \psi}{\partial \tau}+\mathrm{P} \frac{\partial^{2} \psi}{\partial \mathrm{x}^{2}}+\mathrm{Q}|\psi|^{2} \psi=0$

with dispersion and nonlinearity coefficients, $\mathrm{P}$ and $\mathrm{Q}$ respectively, given by the following expressions:

$P=\frac{1}{2} \frac{\partial V_{g}}{\partial k}=-\frac{1}{2 \omega}\left(V_{g}^{2}-u_{0}^{2} \cos (k)\right) ;$

$Q=\frac{3 a \omega}{2}$.

Nowadays, it not secrecy for anybody that the soliton solution of equation (12) depends on the sign of the product $\mathrm{PQ}$. Then we have to analyze the behavior of $\mathrm{PQ}$ in the function of the frequency within the first Brillouin zone. Figure 4 shows the dependence of the dispersion coefficient (13) as a function of the frequency $\left(f_{0} \leq f \leq f_{\max }\right)$ : it appears that $\mathrm{P}>0$ for $f \in\left[f_{0}, f_{s}[\right.$ and $\mathrm{P}<0$ for $\left.f \in] f_{s}, f_{\max }\right]$. There is a particular frequency $f_{s}$ for which $\mathrm{P}=0$. This frequency induces maximum group velocity (see figure 3 ) and imply the resonance of the nonlinearity coefficient $\mathrm{Q}$. The 


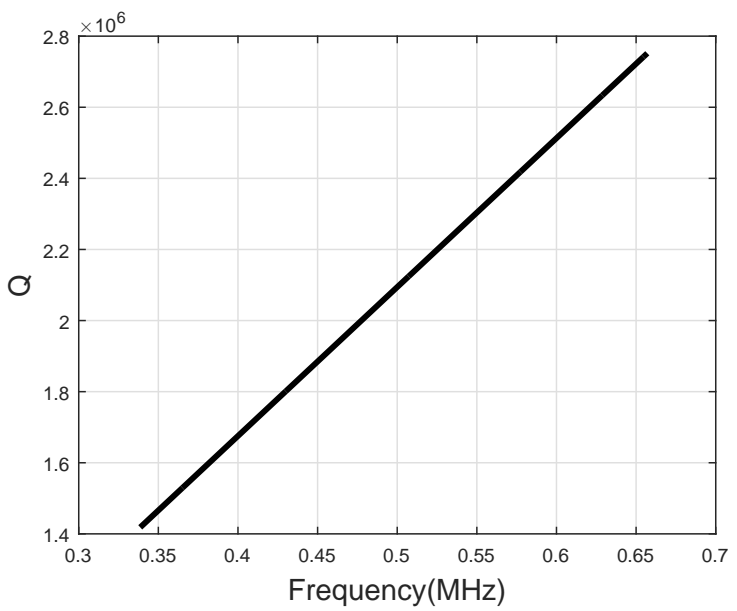

Fig. 5 Nonlinear coefficient (14) as a function of the frequency.

dispersion which generally tends to prevent wave steepening do not exist and equation (12) do not allow soliton solution. The nonlinear coefficient is depicted in figure 5 and it is observed the positive value of $\mathrm{Q}$ in a function of the frequency within the first Brillouin zone. With this observation, one can conclude that the sign of the product $\mathrm{PQ}$ depends exclusively on that of $\mathrm{P}$ as in the vertical dust grain oscillations in dusty plasma crystals [39] and the Schrödinger equation supports following known bright soliton solution [40]

$\psi(\mathrm{x}, \tau)=\alpha \operatorname{sech}[\beta(\mathrm{x}-\mathrm{V} \tau)] \exp (\mathrm{Kx}-\Omega \tau)$,

for $f_{0} \leq f \leq f_{s}$ dark solitons solution

$\psi(\mathrm{x}, \tau)=\alpha \tanh [\beta(\mathrm{x}-\mathrm{V} \tau)] \exp (\mathrm{Kx}-\Omega \tau)$,

for $f_{s} \leq f \leq f_{\max }$. In the equations (15) and (16), $\alpha$ is the soliton's amplitude, $\beta=\alpha \sqrt{\left|\frac{Q}{2 P}\right|}$ is the inverse width of the solitons, $\mathrm{K}$ is the soliton's wave number, $\mathrm{V}$ is the soliton's velocity and $\Omega=(1 / 2)\left(K^{2}-\beta^{2}\right)$ the frequency. Following the solutions (15) and (16), the unknown voltages $V_{n}(t)$ in equation (6) in terms of the original coordinates can be given respectively by:

$\mathrm{V}_{\mathrm{n}}(\mathrm{t}) \simeq \mathrm{V}_{\mathrm{in}} \operatorname{sech}\left[\epsilon \beta\left(\mathrm{n}-\mathrm{V}_{\mathrm{g}} \mathrm{t}\right)\right] \cos \left(\mathrm{kn}-\Omega_{0} \mathrm{t}\right)$,

$\mathrm{V}_{\mathrm{n}}(\mathrm{t}) \simeq \mathrm{V}_{\mathrm{in}} \tanh \left[\epsilon \beta\left(\mathrm{n}-\mathrm{V}_{\mathrm{g}} \mathrm{t}\right)\right] \cos \left(\mathrm{kn}-\Omega_{0} \mathrm{t}\right)$

where $V_{i n}$ is the soliton's amplitude and $\Omega_{0}$ the frequency given respectively by:

$\mathrm{V}_{\text {in }}=2 \epsilon \beta \sqrt{\left|\frac{2 \mathrm{P}}{\mathrm{Q}}\right|}$,

$\Omega_{0}=\omega+2 \epsilon^{2} \Omega P$.

\section{Numerical experiments}

The numerical simulations of this section consist of the integration of the exact discrete equation (6) governing the voltage propagation along the lattice, the local (8)and total energies (8) of the line with the experimental parameters given by Eq. (7). The parameters related to the soliton's amplitude are $\epsilon=0.021$ and $\beta=1$. Figure 2 shows that the frequency is in order of $10^{6} \mathrm{~Hz}$. That means that the integration time is in order of $10^{-6}$ seconde $(\mu s)$. The numerical experimental will be performed with $300 \mu s$ as maximum experimental time.

Any frequency chosen in the phonon band transmission allows obtaining the wave number k using Eq. (9) , the group velocity using Eq. (10), the dispersion coefficient using Eq. (13), the nonlinear coefficient using Eq. (14) and the initial voltage gives by the Eqs. (17) and (18). As found above, the line adopts alternatively RH and LH behaviors. These behaviors will be studied separately in the next sub-sections. For every chosen frequency $\mathrm{f}$ in the pass band $\left(f_{0} \leq f \leq f_{\max }\right)$, the wave vector $\mathrm{k}$ can be determined using the dispersion relation (9) as: $k=2 \arcsin \left(\sqrt{\frac{4 \pi^{2} f^{2}-\omega_{0}^{2}}{4 u_{0}^{2}}}\right)$ and the group velocity using (10). Knowing the frequency, wave vector and the group velocity, it is easy to obtain the value of the dispersion coefficient $\mathrm{P}$ (13) and the nonlinear coefficient Q (14) whose values will be used within the initial conditions.

\subsection{Bright voltage propagation}

It is important to point out on the experimental point of view that bright voltage has been realized with electrical lattice with asymmetric nonlinear capacitor [22] and logarithmic capacitor name as Toda lattice [14]. The initial condition of the bright voltage in our numerical experiment is given by (17).

In figure 6 a), we depict the spatiotemporal contour plots of the discrete voltage of the lattice governed by Eq.(6) for the frequency $\mathrm{f}=0.62 \mathrm{MHz}$ and Eq. (18) (for $\mathrm{t}=0)$ as the initial condition. As our lattice is a conventional transmission line (parallel group and phase velocities ), the wave travel in the right-hand side. One can also observe that the initial pulse maintains its shape, which demonstrates the robustness of the bright voltage soliton solution. Figure $6 \mathrm{~b}$ ) The time evolution of bright voltage in cell 10 . The modulation of the voltage within the time is observed. Its constant amplitude confirms its stability. Figure 6 c) depicts the spatiotemporal propagation of the local energy (8). Firstly, it is evident to observe the propagation of the energy of the 
bright in the right-hand side. This is in agreement with the positive group velocity and the increasing dispersion relation with the wave number. Secondly, there is a localization of energy in the middle of the lattice. This is confirmed by the spatial distribution of the local energy along the line at the integration time $\mathrm{t}=100 \mu \mathrm{s}$ given by figure $6 \mathrm{~d}$ ). The left-handed nonlinear transmission line with Josephson junction [41] has also the localization on the spatial distribution of the local energy observed there but with energy higher than that obtained here.

\subsection{Dark voltage propagation}

For the dark voltage propagation, the initial condition used is Eq. (18) (for $\mathrm{t}=0$ ) for frequency $f_{s} \leq f \leq f_{\max }$. Figure 7 a) shows the spatiotemporal evolution of the dark voltage of Eq. (6) with frequency $\mathrm{f}=0.62 \mathrm{MHz}$. One can see the robustness of the dark voltage within the lattice and the propagation in the right-hand side confirming the fact that our line is the conventional righthanded transmission line. Figure $7 \mathrm{~b}$ ) The evolution of dark voltage in cell 10 . We observe the modulated dark voltage as a function of time. In figure $7 \mathrm{c}$ ), we depict the spatiotemporal evolution of the local energy (8). In agreement with the left-handed nonlinear transmission line with Josephson junction, local energy drop to zero at the soliton centre (see $7 \mathrm{~d})$ ).

\section{Conclusions}

In summary, we have studied analytically and numerically discrete voltage in an electrical lattice with a nonlinear symmetric capacitor. In the linear approximation, the angular frequency increases with the wave number, induces a positive value of the group velocity and predicts the forward waves. By studying analytically the nonlinear lattice using the quasidiscrete approximation, we derived in the small amplitude approximation the nonlinear Schrödinger equation and found that the system supports bright soliton with constant width over time and stable dark soliton. The numerical experiment shows that both voltage and energy travel on the right-hand side. We expect that the results presented in this work may be useful to the enhancement of microwave devices and for phenomenon travelling robust wave.

\section{Conflicts of interest}

The authors declare that there is no conflict of interest.

\section{References}

1. R. Hirota, K. Suzuki, Studies on lattice solitons by using electrical networks, J Phys Soc Jpn. 28, 1366 (1970).

2. R. Hirota, K. Suzuki, Theoretical and experimental studies of lattice solitons in nonlinear lumped networks, Proc IEEE. 61, 1483 (1973).

3. M. Remoissenet, Waves Called Solitons:Concepts and Experiments 3rd Springer-Verlag (1999).

4. T. Dauxois, M. Peyrard, Physics of Solitons, Cambridge University Press, (2005).

5. D. A. Powell, I. V.Shadrivov, Yu S. Kivshar, Asymmetric parametric amplification in nonlinear left-handed transmission lines, Applied Physics Letters 94, 084105(2009).

6. M. Sato, S. Yasui, M. Kimura, T. Hikihara, A. J. Sievers, Management of localized energy in discrete nonlinear transmission lines, EPL 80, 30002 (2007).

7. A. M. Dikande, G-A Bartholomew, Localized short impulses in a nerve model with self-excitable membrane, Phys. Rev. E 80, 041904 (2009).

8. A. Mboussi Nkomidio, S. Noubissie, P.Woafo Dynamics of arrays of legs powered by a discrete electrical model of nerve, Phys. Lett. A 378, 857 (2014).

9. T Kofane, B Michaux, M Remoissenet, Theoretical and experimental studies of diatomic lattice solitons using an electrical transmission line, J. Phys. C: Solid State Phys. 21, 1395 (1988).

10. F. Palmero, L. Q. English, Xuan-Lin Chen, Weilun Li, J. Cuevas-Maraver, P. G. Kevrekidis, Experimental and numerical observation of dark and bright breathers in the band gap of a diatomic electrical lattice, Phys. Rev. E 99, 032206 (2019).

11. L. D. Sekulić, M. V. Satarić, An Improved Electrical Model of Microtubule as Biomolecular Nonlinear Transmission Line, IEEE PROC. 29th Int. Conf. Microelectronics (MIEL2014). doi 10.1109-MIEL.2014.6842098

12. P. Chaurasia, B. K. Kanaujia, S. Dwari and M. K. Khandelwal, Theoretical circuit modeling of tetra bands DNG metamaterial by transmission line theory with very small frequency J. Comput. Electron 20, 1439 (2021)

13. Y. Nejoh, Envelope Soliton of the Electron Plasma Wave in a Nonlinear Transmission Line, Phys. Scr. 31, 415 (1985).

14. P. Marquié, J. M. Bilbault, M. Remoissenet, Observation of nonlinear localized modes in an electrical lattice, Phys. Rev. E 51, 6127 (1995).

15. B. Z. Essimbi, I. V. Barashenkov, Spatially localized voltage oscillations in an electrical lattice, J. Phys. D: Appl. Phys. 35, 1438 (2002).

16. B. Z. Essimbi, T. C. Kofane, Coupling of stationary nonlinear modes in an electrical lattice, Phys. Scr. 76, 480 (2007).

17. A. B. Togueu Motcheyo, C. Tchawoua, M. Siewe Siewe J. D. Tchinang Tchameu, Multisolitons and stability of two hump solitons of upper cutoff mode in discrete electrical transmission line, Phys. Lett. A 375, 1104 (2011).

18. A. B. Togueu Motcheyo, C. Tchawoua, J. D. Tchinang Tchameu, Supratransmission induced by waves collisions in a discrete electrical lattice, Phys. Rew E 88, 040901(R) (2013);

19. A. B. Togueu Motcheyo, C. Tchawoua, M. Siewe Siewe, J. D. Tchinang Tchameu, Supratransmission phenomenon in a discrete electrical lattice with nonlinear dispersion, Commun Nonlinear Sci Numer Simulat 18, 946 (2013).

20. A. B. Togueu Motcheyo, J. D. Tchinang Tchameu, S. I. Fewo, C. Tchawoua, T. C. Kofane, Chameleon' s behavior of modulable nonlinear electrical transmission line, Commun Nonlinear Sci Numer Simulat 53, 22 (2017). 
21. L. Q. English, F. Palmero, A. J. Sievers, P. G. Kevrekidis, D. H. Barnak, Traveling and stationary intrinsic localized modes and their spatial control in electrical lattices, Phys. Rev. E 81, 046605 (2010).

22. P. Marquié, J. M. Bilbault, M. Remoissenet, Observation of envelope and hole solitons in as experimental transmission line, Phys. Rev. E 49, 828 (1994).

23. D. Yemélé, P. Marquié, J. M. Bilbault, Long-time dynamics of modulated waves in a nonlinear discrete LC transmission line, Phys. Rev. E 68, 016605 (2003).

24. S. B. Yamgoué, S. Morfu and P. Marquié, Noise effects on gap wave propagation in a nonlinear discrete LC transmission line, Phys. Rev. E 75, 036211 (2007).

25. L. Q. English, S. G. Wheeler, Y. Shen, G. P. Veldes, N. Whitaker, P. G. Kevrekidis, D. J. Frantzeskakis, Backwardwave propagation and discrete solitons in a left-handed electrical lattice, Phys. Lett. A 375, 1242 (2011).

26. E. Tala-Tebue, D.C. Tsobgni-Fozap, A. Kenfack-Jiotsa, T.C. Kofane, Envelope periodic solutions for a discrete network with the Jacobi elliptic functions and the alternative $\left(\mathrm{G}^{\prime} / \mathrm{G}\right)$-expansion method including the generalized Riccati equation, Eur. Phys. J. Plus 129, 136 (2014).

27. J. E. Ndecfo, G. R. Deffo, S. B. Yamgoué, F. B. Pelap, Alternate backward and forward waves in a coupled nonlinear transmission line, Eur. Phys. J. Plus 135, 57 (2020);

F. B. Pelap, I. Tatsinkou and A. Fomethe, Nonlinear excitations in a continuous bi-inductance electrical line, Phys. Scr. 83, 045009 (2011).

28. C. J. G. Meyers, C. R. Freeze, S. Stemmer, R. A. York, (Ba,Sr)TiO3 tunable capacitors with RF commutation quality factors exceeding 6000, Appl. Phys. Lett. 109, 112902 (2016).

29. Y. Shen, P. G. Kevrekidis, G. P. Veldes, D. J. Frantzeskakis, D. DiMarzio, X. Lan, V. Radisic, From solitons to rogue waves in nonlinear left-handed metamaterials, Phys. Rev. E 95, 032223 (2017).

30. V. Koukouloyannis, P. G. Kevrekidis, G. P. Veldes, D. J. Frantzeskakis, D. DiMarzio, X. Lan, V. Radisic, Bright breathers in nonlinear left-handed metamaterial lattices, Phys. Scr. 93, 025202 (2018).

31. A. B. Togueu Motcheyo, J. E. Macías-Díaz, On the wave transmission in a discrete nonlinear left-handed electrical lattice, Waves in Random and Complex Media (2020) https://doi.org/10.1080/17455030.2020.1859166

32. N. Lazarides, M. Eleftheriou, G. P. Tsironis, Discrete Breathers in Nonlinear Magnetic Metamaterials, Phys. Rev. Lett. 97, 157406 (2006).

33. A. B. Kozyrev, D. W. Van der Weide, Nonlinear lefthanded transmission line metamaterials, J. Phys. D: Appl. Phys. 41173001 (2008).

34. F. Tchier, A. Yusuf, A. Isa Aliyu, M. Inc, Soliton solutions and conservation laws for lossy nonlinear transmission line equation, Superlattices Microstruct. 107320 (2017).

35. H. Halidou, A. Houwe, S. Abbagari, M. Inc, Serge Y. Doka and T. Bouetou Bouetou, Optical and W-shaped bright solitons of the conformable derivative nonlinear differential equation, J. Comput. Electron. 20, 1739(2021).

36. J. Manafian, J. Jalali, A. Ranjbaran, Applications of IBSOM and ETEM for solving a discrete electrical lattice, Opt Quant Electron 49406 (2017).

37. K. Narahara, Collision of nonlinear pulses in travelingwave field effect transistors loaded with Schottky varactors, Journal of Applied Physics 111, 044910 (2012).

38. K. Narahara, Characterization of edge oscillation in a traveling-wave field-effect transistor, Phys. Rev. E 88, 012907 (2013).
39. A. B. Togueu Motcheyo, E. Nkendji Kenkeu, J. Djako, and C. Tchawoua, Backward-wave propagation with vertical dust grain oscillations in dusty plasma crystals, Physics of Plasmas 25, 123701 (2018).

40. Yu. S. Kivshar and G. P. Agrawal, Optical Solitons: From Fibers to Photonic Crystals (Academic, San Diego, 2003).

41. S. Abdoulkary, L. Q. English, A. Mohamadou, Envelope solitons in a left-handed nonlinear transmission line with Josephson junction, Chaos, Solitons and Fractals 85, 44 (2016). 


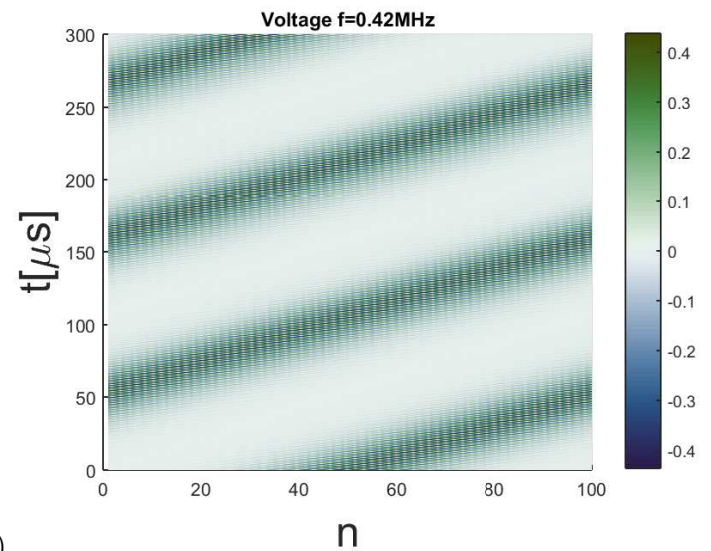

(a)

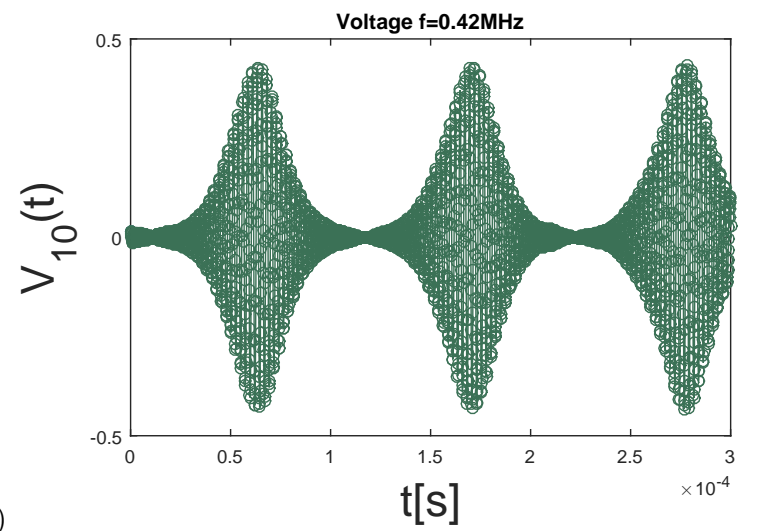

(b)

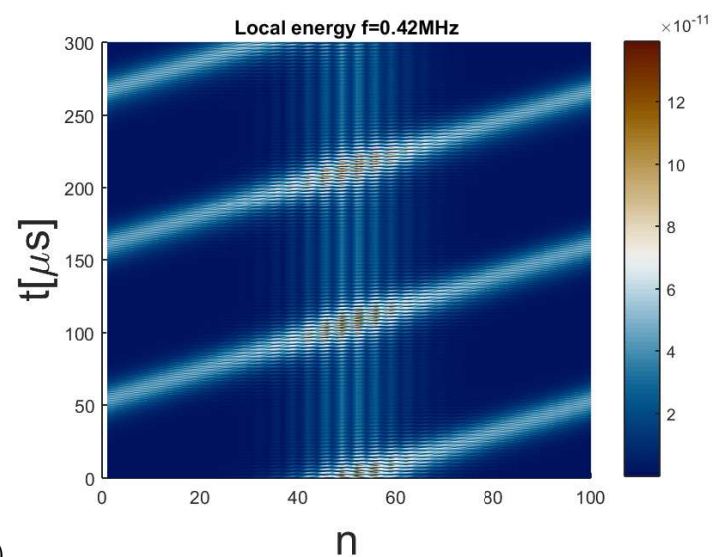

(c)

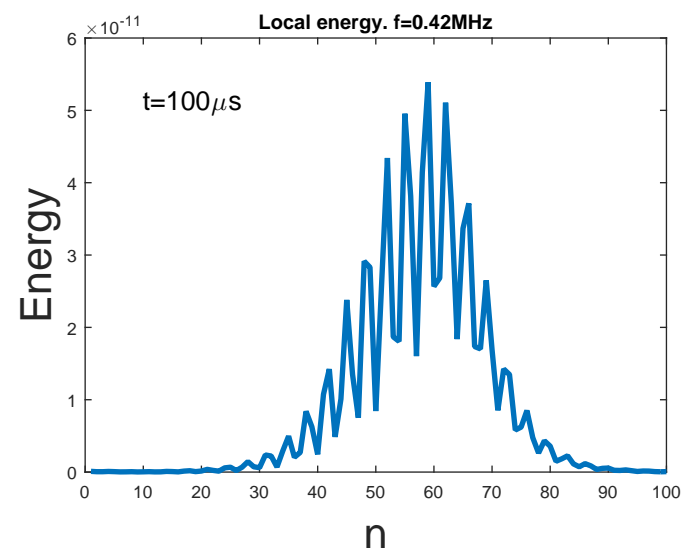

(d)

Fig. 6 a) Spatiotemporal of voltage propagation results the full numerical integration of Eq. (6) for the frequency $\mathrm{f}=0.42 \mathrm{MHz}$ and Eq. (17) (for $\mathrm{t}=0$ ) as the initial condition. b) The evolution of bright voltage in cell 10. c) Spatiotemporal propagation of the local energy (8). d) The energy density of the bright voltage computes at $t=100 \mu \mathrm{s}$.

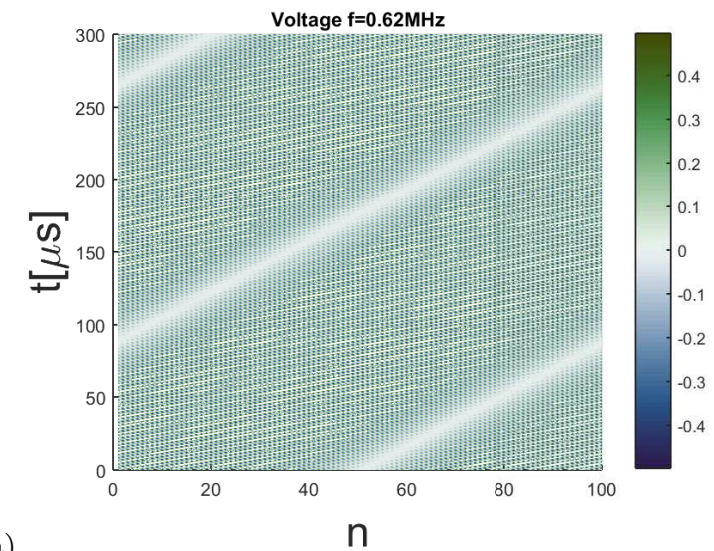

(a)
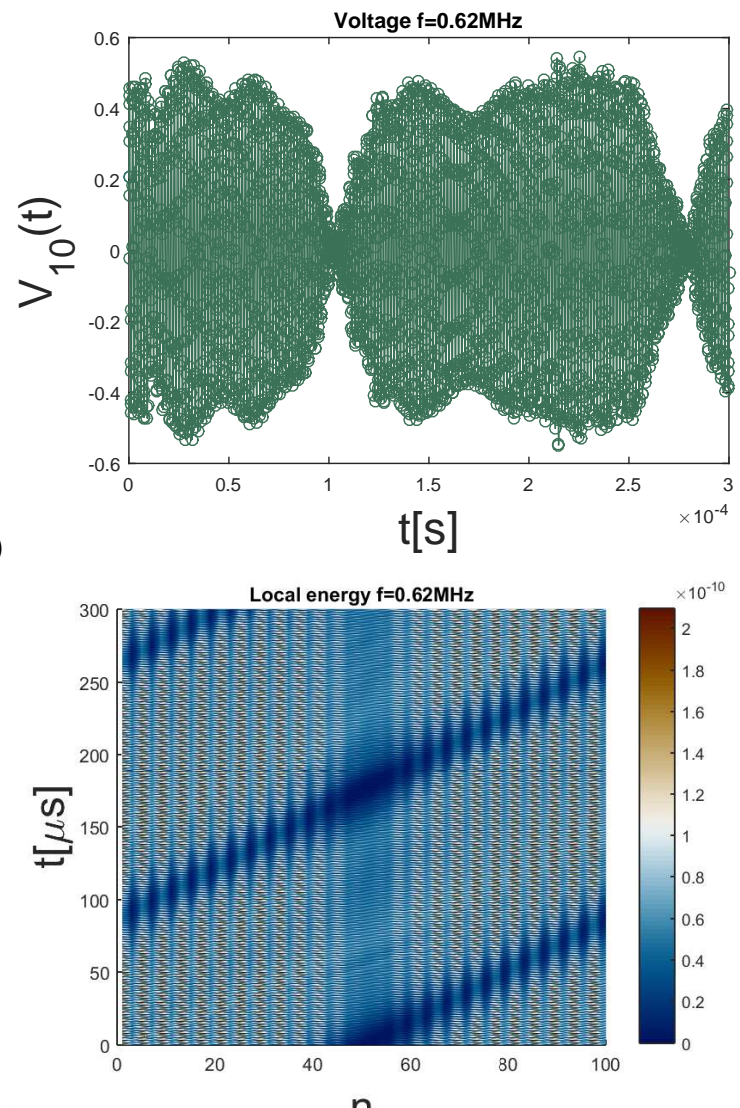

(c)

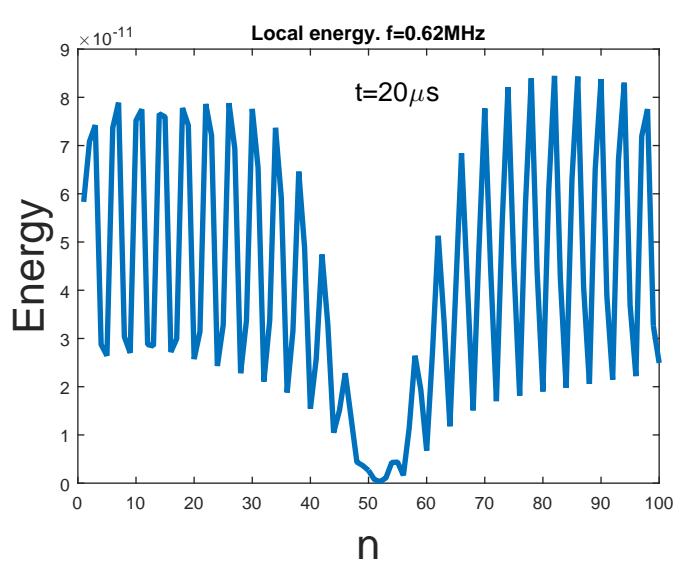

(d)

Fig. 7 a) Spatiotemporal of voltage propagation results the full numerical integration of Eq. (6) for the frequency $\mathrm{f}=0.62 \mathrm{MHz}$ and Eq. $(18)($ for $\mathrm{t}=0)$ as the initial condition. $\mathrm{b}$ ) The evolution of dark voltage in cell 10. c) Spatiotemporal propagation of the local energy (8). d) The energy density of the dark voltage computes at $t=20 \mu \mathrm{s}$. 